\title{
DESCRIPCIONES VACIAS
}

\author{
ALEJANDRO ROSSI \\ Universidad Nacional \\ Autónoma de México
}

Consideremos expresiones como "EI hombre que mide tres metros de altura", "La casa con diez mil puertas", "EI niño que refutó el teorema de Gödel", "La tercera parte del Quijote" y también, claro está, "El actual rey de Francia" y "La montaña de oro". Todas ellas son descripciones definidas, posibles sujetos gramaticales de proposiciones y hablan de individuos u objetos que no existen actualmente. Son expresiones que refieren, pero su referencia es vacía, aun cuando ello se debe a razones contingentes, es decir, es em. píricamente posible que exista un infante que objete con éxito a Gödel, es posible, aunque quizá exagerado, construir una casa con ese número de puertas, no es contradictorio suponer que se hubiera escrito la tercera parte del Quijote. Por consiguiente, la referencia de esas expresiones puede dejar de ser vacia; lo cual indica que una expresión del tipo de las ejemplificadas se refiere a lo mismo ya sea que exista o no exista el individuo u objeto referido. Esto es: si la referencia de las expresiones es vacía por razones no necesarias, entonces lo único que cambia cuando el individuo $u$ objeto existe es el valor de verdad de una proposición como "El actual rey de Francia existe". Pues al decir que es vacía por razones contingentes sin duda estamos afirmando que podría ser el caso que alguna vez existiera aquello mismo a lo cual se refieren - ahora - ese tipo de expresiones. Por tanto es contradictorio suponer que son vacías por razones empíricas y que las expresiones no denotan lo mismo cuando su referencia es vacía y cuando no lo es.

Supongamos, ahora, que con esas expresiones se forman 
las proposiciones "La montaña de oro no existe", "El niño que refutó el teorema de Gödel no existe" y recordemos que a propósito de ellas se ha observado lo siguiente: si son significativas tienen que denotar algo, tienen que referirse a algo, pues de lo contrario serían un flatus, un conjunto de ruidos. Ahora, como la parte de la proposición que denota es la que corresponde a la descripción definida, tal parece que el individuo y objeto denotado no pueden ser más que ese niño prodigio y la montaña de oro. Pero no existen. Luego: si la proposición es significativa debe existir el individuo u objeto denotado, pero entonces las proposiciones "La montaña de oro no existe" y "El niño que refutó el teorema de Gödel no existe" no serían verdaderas sino falsas. Pero sin embargo son verdaderas. Se llegaría así a una situación embarazosa: si se desea explicar la significatividad de las proposiciones es necesario admitir que las descripciones definidas denotan, pero entonces se altera el valor de verdad. No habría manera de explicar cómo es posible que proposi. ciones existenciales negativas sean significativas y, al mismo tiempo, verdaderas. La única forma de conciliar la significación de estas proposiciones con sus valores de verdad consistiría en aceptar (o en reconocer) que las descripciones definidas "La montaña de oro" y "El niño que refutó el teorema de Gödel" denotan objetos e individuos que tienen un tipo de existencia distinta a la espacio-temporal: ser, subsistencia, existencia lógica, ideal, etc. Si se acepta, la descripción definida de la proposición "La montaña de oro no existe" denota un objeto con ser o con subsistencia - del cual se predica que no existe espacio-temporalmente- salvándose, así, la significación y manteniéndose el valor de verdad original. Las nuevas entidades garantizarian la sig. nificación de esa clase de proposiciones.

En relación a este planteamiento conviene, en primer lugar, recalcar la conexión estrechísima que allí se establece entre las entidades referidas y la significación de la descripción definida y, en consecuencia, de la proposición. Tan 
directa es la dependencia que si no se postula una entidad denotada por la descripción definida, ésta no se distinguiría de un flatus vocis. Lo cual querría decir que la existencia de la entidad denotada es necesaria para que la proposición en cuestión tenga la significación que en efecto posee. $O$ con mayor generalidad: la significación de la proposición está fijada, de alguna manera, por el objeto denotado. Pues precisamente en esa premisa se basa la posible fuerza del razonamiento que concluye en la aceptación de entes subsistentes. En segundo lugar, es menester prestar atención a algo al parecer muy simple, a saber, que en dicho planteamiento se maneja un tipo de ejemplos a diferencia de otros. Esto es: la necesidad de postular una clase de entes se plantea a propósito de ciertas proposiciones, lo cual indica que se está entendiendo el término significación en la acepción según la cual es correcto decir que "La montaña de oro no existe" es una proposición cuya significación es distinta a la de "El niño que refutó el teorema de Gödel no existe". Se trata de la acepción según la cual explicar el significado siempre es explicar el significado de una determinada proposición; en suma, es la acepción que usamos cuando afirmamos que una proposición en castellano significa lo mismo que una determinada proposición expresada en otro lenguaje. Esta forma de emplear el término equivale más o menos a lo que entendemos por 'contenido' de una proposición. ${ }^{1}$ Que en el planteamiento anterior se está usando 'significación' como equivalente a 'contenido' de una proposición se nota, además, en que una vez que se ha decidido acerca de la necesidad de postular una entidad, no se propone -digámoslo así- una entidad en general, sino aquella que, según los casos, responde al 'contenido' de la proposición: la montaña de oro o el niño que refutó a Gödel. Así, pues, la nueva entidad pretende 'salvar' la significación - contenido- de esta proposición y sería entonces absurdo

1 Quede claro que aquí sólo queremos, y de una manera informal, identificar esa acepción; no intentaremos ni definirla ni analizarla. 
que lo hiciera proponiendo la existencia ideal o la subsistencia de una entidad cualquiera.

Tal vez sea útil ahora introducir la expresión 'significación normal'. 'Significación' se toma en el sentido ya descrito y por 'significación normal' habrá que entender, simplemente, el contenido que una proposición de hecho tiene -o quiere dársele- en un determinado contexto discursivo. Así, por ejemplo, si una persona afirma "La montaña de oro no existe" y sostiene que es verdadera porque espacio. temporalmente no existe una montaña con esas características, diremos que la 'significación normal' de esa proposición consiste en referirse de una determinada manera a una montaña de oro espacio-temporal. E igual en relación a las demás proposiciones mencionadas.

Respecto a nuestro problema nos encontraríamos, entonces, ante esta situación:

(a) que la 'significación' de una proposición no puede conceptuarse, o explicarse, sin la aceptación de la existencia, o subsistencia, de una entidad;

(b) que el problema, sin duda alguna, se plantea a partir de la 'significación normal' de determinadas proposiciones. La 'significación normal' de un tipo de proposiciones es el dato que genera el planteamiento que estamos examinando.

Ahora bien, de (a) y (b) se concluye fácilmente que

(c) los entes subsistentes o ideales se introducen para salvar la 'significación normal' de la proposición en cuestión. En efecto: de lo que se trataba, en principio, era de ver cómo es posible que una proposición como "La montaña de oro no existe" pueda tener la 'significación normal' que de hecho tiene cuando es ella misma la que parece exigir que no exista el ente al cual se refiere. Son las 'significaciones normales' de esa clase de proposiciones las que crean 
un problema conceptual y se busca, entonces, dar con una solución que preserve la 'significación normal' de la proposición $y$, al mismo tiempo, no entre en Sin embargo, conflicto con lo que se afirma en (a).

(d) si se trata, como ocurre aquí, de la 'significación normal', entonces ésta requiere un determinado ente - objeto o individuo- y no, según dijimos líneas atrás, cualquier ente individual; cuál sea ese ente se deduce, en cada caso, de cuál sea la 'significación normal' de la proposición. Repetimos: se intenta 'salvar' la 'significación normal' de una proposición específica y, por consiguiente, la entidad que se supone necesaria debe, forzosamente, corresponder al contenido de la proposición.

Tenemos, pues, que lo que se requiere para explicar y garantizar la significación normal es un ente determinado. Por tanto, si se propone un ente que no corresponde a la significación normal de la proposición, no se está ni explicando ni 'salvando' dicha significación normal. Más aún, ¿qué ocurriría si se introdujera otro tipo de entidad? La respuesta debe ser ahora obvia: se alteraría la significación normal de la proposición. En efecto, de una proposición que se refiere, de una determinada manera, a una montaña de oro espacio-temporal se pasa a una proposición que habla de una montaña de oro ideal, o subsistente, o lógica. Y sea lo que fuere esta proposición, se analice de éste o de aquél modo, se trata - sería difícil negarlo- de una proposición diferente a la original. Si a una persona que afirma la verdad de "La montaña de oro no existe" dando como razón la de que en el mundo espacio-temporal no se encuentra una cosa así, se le insinuara que la 'significación' de la proposición consiste en hablar, de una determinada manera, acerca de una montaña de oro ideal - dicha persona, qué duda cabe, reaccionaría negativamente: no afirmó esa proposición 
sino la otra. Se necesitaría mucha teoría, o pseudo teoría, para hacerle cambiar de opinión. Vemos entonces que si se postula un ente distinto al que exige la 'significación normal', el resultado es que cuando se afirma una proposición cuyo sujeto no existe espacio-temporalmente siempre estaríamos hablando de otra cosa; lo cual es equivalente a sostener que estaríamos afirmando otra proposición, con una 'significación normal' diferente. La postulación de entes ideales o lógicos cuando más 'salvaría' el conjunto de signos o sonidos que configuran la proposición -en el sentido de que tendrían una significación- pero de ninguna manera salvaría la significación normal que se pretendía garantizar. En suma, la solución propuesta se aplica a una acepción tal de significación que el cambio de ente la altera y la transforma: la significación normal no tolera la indeterminación ontológica.

Si lo anterior es correcto, estamos frente a un ejemplar auténtico de razonamiento vicioso. Las significaciones normales de un tipo de proposiciones plantean, al parecer, un problema, pero la forma de resolverlo no respeta los datos que lo originan: los entes subsistentes o lógicos se introducen para salvaguardar la significación normal de una proposición dada $\mathrm{y}$, sin embargo, esas entidades no explican cómo es posible que esa proposición tenga esa significación normal -porque necesariamente se genera otra proposición. Ampliar los límites de lo existente es, en este caso, una tarea inútil.

Dijimos, además, que las expresiones que funcionan como sujetos gramaticales de las proposiciones que estamos examinando son vacías por razones empíricas y este hecho revela, aún con mayor fuerza, la confusión que se crea al recurrir a los entes ideales. En efecto: si esa es la razón por la cual son vacías, entonces deben referirse a lo mismo que en el caso en que no fueran vacías. Sin embargo, de acuerdo con la interpretación criticada, en la proposición "La montaña de oro no existe", la descripción definida no tiene la 
misma referencia que la que posee en "La montaña de oro existe" si esta proposición fuera verdadera. Y la consecuencia inmediata sería que "La montaña de oro no existe" no es la negación de "La montaña de oro existe". Se trataría de dos proposiciones diferentes: una diría que la montaña de oro subsistente no existe espacio-temporalmente y la otra que espacio-temporalmente existe una montaña así y por lo tanto no hay motivo para ver la primera como la negación de la segunda. Ahora bien, de la verdad de la proposición "La montaña de oro existe" se sigue la verdad de la proposición “'La montaña de oro' no es vacía". Pari passu, de la verdad de "La montaña de oro no existe" se sigue la verdad de " "La montaña de oro' es vacía". Pero si "La montaña de oro no existe" no es la negación de "La montaña de oro existe", entonces "La montaña de oro' es vacía" sin duda es una proposición siempre falsa. Sin embargo, si es siempre falsa, no es el caso entonces que estas descripciones definidas sean del tipo de aquellas que pueden ser vacías. Y esto equivale, claro está, a la alteración de ciertos datos del problema, es decir, la introducción de nuevas entidades es incompatible con la caracterización de estas expresiones como siendo vacías por razones contingentes. $\mathrm{Y}$, no obstante, las dificultades se formulan justamente en base a esas características, ya que las significaciones normales de estas proposiciones implican que el objeto o individuo referido por la descripción definida puede no existir. En el caso de "La montaña de oro no existe" se está afirmando, en efecto, que no hay tal objeto y, a fortiori, que la expresión "La montaña de oro" puede ser vacía. Negarlo es incurrir, nuevamente, en un razonamiento vicioso.

De lo anterior se desprende que la alteración de las significaciones normales de esta clase de proposiciones se produce cualquiera que sea el tipo de las nuevas entidades propuestas. Poco importa, para mencionar los dos casos extremos, que el ente que se introduzca sea una idea subjectiva o un objeto con existencia $\longrightarrow$ subsistencia- extra mente: 
se elija el platonismo abierto o su contrario, el resultado es el mismo. Pues la significación normal supone un ente determinado y cualquier cambio la afecta. Si el nuevo ente fuese una 'idea', notemos una vez más que una proposición que habla de la idea que alguien tiene de la montaña de oro, posee una significación normal diferente a la de la proposición que pretende afirmar algo de la montaña de oro espacio-temporal. Lo cual es inevitable dado el planteamiento.

Examinemos, ahora, una variante del razonamiento que desemboca en los entes ideales. Se admitiría la necesidad de acudir a un lenguaje de objetos en la explicación de la significación: uno de sus elementos básicos, sobre todo si se trata de expresiones referenciales, es la relación de un signo, o de un conjunto de signos, con algo, con un objeto o individuo. No parece ser posible hablar de la significación de unos signos sin introducir la conexión con entidades, sin acudir a un lenguaje 'objetivo'. De no hacerlo, se imposibilita la distinción entre una palabra, o una expresión, y un flatus. Pero ocurre que hay expresiones referenciales vacias, más aún, se encuentran en el lenguaje expresiones y proposiciones que por la naturaleza del caso no pueden dejar de serlo y, en consecuencia, no es posible afirmar la existencia de aquello a lo cual se refieren sin incurrir en contradicciones. Estos hechos lingüísticos obligarían a modificar la conexión entre significación y entidades y a concebir estas últimas -indispensables en la explicación de las significaciones - como 'objetos intencionales', esto es, como una especie de representaciones mentales que permitirían saber de qué está hablando la proposición o a qué se refiere la expresión en cuestión. Los objetos intencionales configurarían el 'contenido' de la proposición cuyo sujeto es una descripción definida y permitirían mantener la conexión necesaria aun cuando la descripción definida fuese vacía: mientras se dé una relación entre esas representaciones mentales y los signos lingüísticos, la significación de la propo- 
sición está salvada. Esto mismo suele expresarse diciendo que no debe confundirse la significación, o el sentido, de una proposición con su referencia: cuando la proposición es vacía, falla la referencia, pero la significación o el sentido permanece. Sin embargo es importante darse cuenta de que por sí misma esta conclusión no añade gran cosa al planteamiento anterior puesto que allí se partía justamente de ese dato, a saber, que la proposición es significativa aun cuando no existe el objeto o individuo al cual se refiere: $e x$ hypothesis, la proposición con referencia vacía es significativa. La novedad tal vez reside en la terminología. Lo que realmente interesa es la explicación que se propone de la permanencia del sentido o de la significación, o sea, vía objetos intencionales. Ahora bien, el 'sentido' que se intenta garantizar con los objetos intencionales no es otra cosa que la 'significación normal' de una proposición dada; pero si el sentido es igual a significación normal, entonces la intromisión de los objetos intencionales precipita el resultado conocido: la alteración de la significación normal o del sentido de la proposición. Una vez más parece imposible poder sostener que el sentido necesariamente requiere la conexión con entidades y al mismo tiempo que estamos hablando de la significación normal de una proposición como "La montaña de oro no existe"; no es posible porque, según sabemos, 'sentido' en esta acepción exige que el objeto que lo determina sea el objeto acerca del cual se habla, v.gr. la montaña de oro espacio-temporal. Luego, si sentido es igual a significación normal, el objeto intencional no puede solucionar el problema cuando la referencia de la proposición es vacía. Estaríamos nuevamente cambiando los términos del problema; partimos de una proposición cuyas características semánticas nos plantean ciertas dificultades y proponemos una solución que da por resultado otra proposición.

Consideremos, ahora, una posible interpretación de la 'variante', la cual, si fuera válida, nos obligaría a modifi- 
car nuestras conclusiones. Consistiría, básicamente, en insistir en que al objeto intencional no debe concedérsele ninguna clase de existencia, ni siquiera la ideal; y que, por consiguiente, la conexión no es entre un algo (signo) y otro algo (entidad). Si la relación no se concibe así, se elimina el fundamento para hablar de otro ente que vendría a ocupar el lugar de aquél del cual se habla en la 'significación normal' de la proposición. Si sólo nos atenemos a las expresiones y proposiciones referenciales, diremos entonces que el término 'objeto intencional' lejos de denotar a un ente determinado, trata de describir el rasgo esencial de una expresión, o sea la dirección hacia algo distinto de ella, siendo secundario que exista o no exista ese algo. Objeto intencional debería entenderse de la siguiente manera: no como un objeto especial sino como la tendencia hacia un objeto propia de cualquier expresión. No se trata, entonces, de que haya una expresión referencial y, además, un objeto intencional - esta sería una interpretación inadmisible- sino sólo una expresión que pretende hablar de un determinado objeto. ¿Cuál sería en el caso de "La montaña de oro"?: la montaña de oro espacio-temporal. En consecuencia: si el sentido se constituye en la intencionalidad hacia un objeto y éste es el propio de cada significación normal, es posible explicar, sin alteración alguna, el sentido de una proposición con referencia vacía.

Dada, pues, una expresión como "El actual rey de Francia" o como "La montaña de oro", se dice que sus 'sentidos' o sus significaciones normales consisten en la dirección hacia un objeto, en su intención hacia algo distinto del signo: dada una expresión y la posible proposición de la cual es su sujeto gramatical, sus significaciones normales tienen siempre que explicarse en función de un objeto. Entonces parece natural concluir que la explicación de sus 'significaciones normales' supone, de alguna manera, la admisión de ese objeto; pues si no fuese así no podría determinarse cuál es su sentido o su 'significación normal'. Pero ¿cuál es ese 
objeto en el caso de "El actual rey de Francia"? La res. puesta es clara: el actual rey de Francia. Pero, sin duda, el actual rey de Francia no existe. Luego, si en este esquema de explicación no se incluye el objeto de la intención es im. posible determinar y aclarar el significado de la proposición. El esquema debe asumir, como lo prueba el ejemplo anterior, objetos que no existen. Ahora bien, si el objeto al cual tiende la proposición y que especifica cuál es su significado es un objeto que no existe, entonces su significación normal se altera. En efecto, habría que explicar la significación normal de la expresión o de la proposición como hablando o como refiriéndose a un objeto inexistente precisamente en aquellos casos en que esas expresiones se usan para referirse a personajes de carne y hueso y a montañas espacio-temporales. Si se afirma que el objeto de la intención explica y determina el 'contenido' de la expresión y resulta que dicho objeto no existe, el contenido debe alterarse. Si se observa que aquí en realidad no se está afirmando la existencia de unos objetos o individuos, que no se está ni descubriendo, ni reconociendo su existencia sino, más bien, asumiendo ciertos elementos explicativos, la respuesta es que entre esta postura y la que de una manera más directa y menos matizada acepta la necesidad de la postulación de entes subsistentes no hay, a nuestro entender, una diferencia de principio. Lo esencial en uno y en otro caso es la recurrencia al 'objeto' para explicar las 'significaciones normales'; la diferencia en cuanto al status de ellos - si son subsistentes o son simples elementos de esquemas interpretativos - es asunto menor en comparación con la necesidad de asumirlos. Y esa es la causa de que tampoco según esta variante podamos aclarar cómo es posible hablar de lo que no existe conservando la proposición su 'significación normal'.

Para no incurrir en las dificultades anteriores podría proponerse, cuando se utiliza la pareja 'sentido-referencia', eliminar del 'sentido' la llamada 'dirección objetiva' o, en otros 
términos, el 'aspecto referencial'. Se eliminaría de un tajo la base para plantear la alteración de la significación normal de la proposición. Adviértase, sin embargo, que el resultado no sería aceptable ya que el 'aspecto referencial' es el que permite saber de qué está hablando la proposición. Porque es evidente que, por un lado, la significación normal de este tipo de expresiones incluye como parte esencial el referirse a un objeto o individuo y que, por otro lado, ese aspecto de la significación normal corresponde, en este es. quema conceptual, a la 'dirección objetiva', a la 'intencionalidad'. Si ésta se separa nos quedamos con una acepción de 'sentido' más o menos equivalente a 'manera de decir' o 'forma de hablar'. Pero es claro, entonces, que nos hemos alejado mucho del dato del cual partíamos, a saber, la significación normal, pues ésta, sin ningún género de dudas, se encuentra mutilada si además de la 'forma de hablar' no se incluye la alusión a aquello de lo cual habla precisamente de ese modo. Nos encontraríamos ante una nueva forma de alteración de los datos del problema original: comenzaríamos con una determinada proposición y terminaríamos, para evitar la intromisión de objetos, con un fragmento de ella. Nótese, además, que si se trabaja con esta acepción de sentido, la distinción 'sentido-referencia' es inaplicable a nuestra situación. En efecto, si el propósito de la distinción es salvar el sentido cuando la referencia es vacía, pero por sentido se entiende únicamente la 'manera de decir', lo que queda cuando no existe el ente referido no es lo que debería salvarse, esto es, la significación normal en sus dos aspectos. En resumen, si se ofrece una versión del 'sentido' en términos de un 'lenguaje objetivo', se genera otra significación normal; si, por el contrario, se abandona el aspecto referencial, se asume una acepción de 'sentido' muy extraña, según la cual éste no guarda relación alguna con aquello de lo cual se habla y, por tanto, no puede verse como la 'sig. nificación normal'. Si se da cuenta del aspecto referencial hay que aceptar - con todas las reservas que se quieran- 
ciertos objetos, pero entonces se produce la alteración; si ésta se evita, no sabríamos de qué estamos hablando.

Para terminar con este punto recordemos que a veces, en otros contextos de problemas, 'sentido' o 'significación' se conciben sin el aspecto referencial; cuando dos proposiciones, por ejemplo, se refieren a lo mismo pero en forma distinta parece que la manera correcta de describir la situación es hablando de sentidos diferentes y aspectos referenciales iguales. Se trataría de una distinción que se llevaría a cabo dentro de la unidad que es la 'significación normal'; de ninguna manera es la distinción general entre 'significación' o 'sentido' y el objeto o individuo referido. Cuando dos proposiciones hablan de manera distinta de un mismo objeto que no existe, el aspecto referencial permitiría concluir que el objeto del cual se habla es el mismo. De nuevo estamos frente a un sentido alejado del concepto de significación normal e inconsistente con la acepción que debe dársele si, por otra parte, se quiere aplicar a nuestro problema la distinción 'sentido-referencia'. Si se arguye que se trata precisamente de una distinción establecida dentro de la significación normal de la proposición y que, por tanto, es suficiente cierto ajuste terminológico para despejar el equívoco - no empleando 'sentido' en los dos casos y reservando el término para la 'significación' que abarca el aspecto referencialentonces simplemente se nos está proponiendo volver a las dificultades anteriores. ${ }^{2}$

Como conclusión quisiéramos hacer dos observaciones. En primer lugar recalcar que la solución que recurre a otros entes - ya sea en una versión platónica franca o en una versión más encubierta - no es defectuosa sólo porque nos obliga a aceptar nuevas entidades, pues ello implicaría que éstas permiten resolver de alguna manera el problema. Tampoco se trata de que estemos frente a una posible solu-

2 Para algunas dificultades en Husserl relativas a estas distinciones, léase nuestro trabajo "Sentido y sinsentido en las Investigaciones Lógicas", Diánoia, F.C.E. México, 1960. 
ción cuyo origen esté en una tesis equivocada acerca de la significación de las descripciones definidas - confundirlas con nombres, por ejemplo. Porque en este caso estaríamos nuevamente admitiendo su plausibilidad. El defecto, a nuestro modo de ver, es más grave: si recurrimos a nuevos entes no llegamos a una solución posible, a una teoría más o menos comprensible, sino que la solución y la teoría no son ni siquiera planteables. En segundo lugar, hay que notar que la alteración de la significación normal sobreviene porque se supone que la significación de una expresión implica necesariamente la asunción del objeto o individuo del cual habla. En otras palabras: el conflicto se crea cuando se interpreta la primera premisa - - necesidad de entes- en términos de la significación normal, como si esa premisa hablara de las significaciones normales. Sin embargo, si nos preguntamos de dónde viene esa premisa, de dónde extrae su fuerza, no podemos responder que se basa en alguna observación relativa a las significaciones normales; pues las significaciones normales de este tipo de proposiciones pueden perfectamente ser vacías y significativas; éste es, justamente, uno de los datos del problema. Si nos fijamos sólo en ellas es imposible llegar a la primera premisa. Más aún, la explicación que se sugiere es opuesta. Entonces ¿en dónde se origina esa tesis? Nuestra sospecha, que no analizaremos aquí a fondo, es que se apoya en una consideración muy general acerca de las palabras: en la constatación de lo que podríamos llamar la Transitividad de todo signo lingüístico. Es decir, en la idea de que un determinado signo es una palabra, o un elemento del lenguaje, en la medida en que es un símbolo de algo. Se dice entonces: la significación de una palabra consiste en su conexión con algo del cual es, precisamente, el símbolo. Pero con ello no se sostendría que todas las palabras deben interpretarse como substantivos o como nombres, sino simplemente se querría decir que las palabras son símbolos $-\mathrm{y}$ asi se entiende que se establezca una oposición entre palabra y flatus. Se trataría de una 
especie de definición de 'palabra'. Luego vendrán las distinciones entre los diferentes usos de ellas. Ahora bien, si esta es la zona en donde la primera premisa puede resultar plausible y hasta válida, también es verdad que allí se maneja una acepción de significación que no guarda relación alguna con la significación normal. Esta última puede ser vacía sin que deje de ser cierta la tesis acerca de la naturaleza simbólica de la expresión o de las palabras que la componen. No hay paralelismo. Si se deslindan los campos no cabe el planteamiento del problema, no cabe exigir la postulación de una entidad denotada por "El actual rey de Francia". La primera premisa sólo nos obligaría a reconocer el carácter transitivo de ese signo complejo; pero no nos fuerza ni a admitir nuevos entes ni a explicar siempre el contenido de la expresión en términos de objetos individuales. 
Expressions such as "The man who is ten feet tall", "The house with ten thousand doors", "The third part of Don Quijote", "The child who refuted Gödel's theorem", and of course "The golden mountain" and "The present King of France", are definite descriptions that speak of individuals or objects which do not actually exist; they are expressions that refer, but their reference is empty, although this is due to contingent reasons. Thus, the reference of these expressions can cease to be empty: for when we say that it is empty on account of reasons which are not necessary, we are affirming that it could be the case that at some time there might exist the object to which they are now referring. It is contradictory to suppose that these expressions are empty because of empirical reasons and that they do not denote the same thing when their reference is empty and when it is not.

Concerning propositions such as "The golden mountain does not exist", "The child who refuted Gödel's theorem does not exist", it has been observed that if they are meaningfull, they have to denote something: if they don't, they would be a flatus. Then, if the proposition has a meaning, the denoted object would have to exist; but then the proposition would not be true, but false. However, it is true. An embarrasing situation would thus arise: there would be no way of explaining how it is possible that negative existential propositions could be meaningfull and true. The solution would be to accept the fact that they denote subsistent objects or individuals, or objects with ideal or logical existence: the definite description of the proposition "The golden mountain does not exist" would denote a subsistent object from which it is predicated that it does not exist spatio-temporally -thus saving its meaning and maintaining the original truth-value. The new entities would guarantee the meaning of that class of propositions.

In what is put forth, a very close connection is established between referred entities and the meaning of the definite description: the meaning would be fixed by the denoted object. Furthermore, the term 'meaning' is being understood in the sense according to which it is correct to say that "The golden mountain does not exist" is a proposition whose meaning is different from "The child who refuted Gödel's theorem does not exist"; it is the sense that we use when we affirm that a proposition in spanish means the same 
as a certain proposition expressed in another language. It is that which is more or less equivalent to what we understand by the 'content' of a proposition. We can see that 'meaning' is being used in that way, in the fact that, once it is decided to postulate an entity, it is not proposed any entity, but that which according to the cases, responds to the content of the proposition: The golden mountain, the child who refuted Gödel's theorem.

The expression 'normal meaning' is introduced. 'Meaning' is taken in the described sense, and by 'normal meaning' would have to be understood simply the content that a proposition, in fact, has, or one wants to give it in a determined discursive context. If someone affirms "The golden mountain does not exist", and he maintains that this is true because spatio-temporally such a mountain does not exist, we will say that its normal meaning consists in referring in a determined manner to a spatio-temporal mountain.

In respect to our problem, we will find ourselves confronted with the following situation:

(a) that the 'meaning' of a proposition cannot be explained without accepting the existence, or subsistence, of an entity;

(b) that the problem, without any doubt, comes out apropos of the 'normal meaning' of certains propositions. The 'normal meaning' of certain types of propositions is the datum which generates the problem.

Now then, from (a) and (b) it can easily be concluded that:

(c) the subsistent or ideal entities are introduced in order to save the 'normal meaning' of the proposition in question. In fact, the question was to see how it was possible that a proposition such as "The golden mountain does not exist" could have the 'normal meaning' that actually has when it is the 'normal meaning' itself that seems to demand that the entity to which it refers does not exist. The normal meanings of these propositions are ones which create a conceptual problem; so we try to find a solution which wouId preserve the 'normal meaning' and would not come into conflict with what has been affirmed in (a).

However,

(d) if, as it happens here, we are dealing with the 'normal meaning', then this requires a determined object or entitv or individual, - and not, as it has been said, any individual entity. If it is intented to 'save' the normal meaning of $a$ specific proposition, the entity which is supposed to be necessary, must correspond, necessarily, to the content of the proposition. 
So, if an entity is proposed which does not correspond to the normal meaning of the proposition, it does not explain or save it. Even more: the normal meaning would be altered. In fact, a proposition which refers in a determined way to spatio-temporal mountain, passes into a proposition which speaks of an ideal, logical, or subsistent golden mountain. And, no matter what this proposition would be, no matter how it might be analysed, it would doubtless be a different proposition. Then, if a different entity is postulated to that which requires the normal meaning, the result would be that when a proposition is affirmed whose subject does not exist spatio-temporally, we would always be speaking of another thing. Briefly, the proposed solution applies to such a sense of 'meaning' that the change of the entity will alter and transform it: normal meaning does not tolerate ontological indetermination.

If this is correct, we are faced with a vicious reasoning. The normal meanings of a certain type of propositions present a problem, but the form of resolving it does not pay respect to the data that originated it: the subsistent entities are introduced in order to save normal meaning, however, they do not explain how is it possible that that proposition could have that normal meaning -because another proposition is necessarily generated.

Furthermore, if these expressions are empty because of empirical reasons, they must refer to the same thing to which they would refer if they were not empty. But according with the criticized interpretation, in the proposition "The golden mountain does not exist", the definite description does not have the same reference which has in "The golden mountain exists", if the later were true. The consequence would be that "The golden mountain does not exist" is not the negation of "The golden mountain exists": one proposition would say that the subsistent golden mountain does not exist spatio-temporally, and the other that spatio-temporally there exists such a mountain; there is no motive for viewing the first as the negation of the second. Now, from the truth of the proposition "The golden mountain exists" follows the truth of the proposition "'The golden mountain' is not empty". Pari passu, from the truth of "The golden mountain does not exist" follows the truth of "The golden mountain' is empty". But if "The golden mountain does not exist" is not the negation of "The golden mountain exists", then "The golden mountain' is empty" is doubtless a proposition which is always false. But if it always false, it is not the case that these definite descriptions would be of the type of those that might be empty. And this is equivalent to the alteration of certain data of the problem: the introduction of new 
entities is incompatible with the characterization of these expressions as being empty because of contingent reasons. And nevertheless, the difficulties are formulated in the basis of those characteristics, since the normal meanings imply that the object or individual referred by the definite description might not exist. In the case of "The golden mountain does not exist" what is being affirmed is that there is no such object, and, a fortiori, that the expression "The golden mountain" might be empty. To deny it is to fall again into a vicious reasoning.

From what has been said before it can be deduced that the alteration of the meaning of this class of propositions is produced whatever the type of the new entities may be. It is of little import that open Platonism or its contrary is chosen: the result is the same, since the normal meaning supposes a determined entity and any change af. fects it.

Then it is examined, in its different aspects, a variation of the former problem, that is, that which recurs to 'intentional objects', and it is concluded that there is also a changing of the terms of the problem: it starts with a proposition whose semantic characteristics involve certain difficulties and proposes a solution that yields, as a result, another proposition.

As a conclusion, two observations may be made. In the first place, we insist that the solution which recurs to ideal entities it is not only defective because it obliges us to accept them, since that would imply that these entities could resolve the problem in some manner. The fault is deeper: if new entities are postulated, we don't get a possible solution or a more or less comprehensible theory, but both the solution and the theory are not even conceivable. In the second place, it is noted that the alteration of the meaning overcomes when the first premise - necessity of entitiesis interpreted in terms of the normal meaning, as if that premise would speak of the normal meaning. However, if we ask ourselves from where does that premise derives its force, we see that it is not based in some observation relative to the normal meanings of these propositions, since they can be empty and have a meaning; this is, precisely, one of the data of the problem. If we only pay attention to those it is impossible to reach the first premise. Furthermore, the explanation suggested is the opposed one. Then, from where does that thesis originate? Our suspicion, that we will not analyze in detail, is that it is founded in a very general consideration concerning words: in what we could call the Transitivity of all linguistic signs. That is to say, the idea that a certain sign is a word, or is an element of language, in the measure in which 
it is a symbol of something. It is then said: the meaning of a word consists in its connection with something, of which it is precisely the symbol. But with that it is not mantained that all words must be interpreted as substantives or as names, but it would simply amount to say that words are symbols -and so is understandable the opposition drawn between a word and a flatus. It would be a kind of definition of 'word'. And then distinctions between the different uses of words may be made. Now then, if this is the area where the first premise may result plausible or even valid, it is true also that we are dealing there with a sense of 'meaning' that does not keep any resemblance with the normal meaning. This last can be empty without ceasing to be true the thesis concerning the symbolic nature of the expressions or of the words which compose it. There is no parallelism. If we distinguish between them, the problem does not arise, and there is no ground for the postulation of an entity denoted by "The present King of France". The first premise would only compel us to recognice the transitive character of that complex sign; but it would not force us to admit new entities, nor to always explain the content of the expression in terms of individual objects. 